\title{
Finding prodromal frailty in a community-dwelling healthy older cohort by survey of BDNF or hand grip strength classified by BMI
}

Mitsugu Hachisu, ${ }^{\mathrm{a}, \mathrm{b}^{*}}$, Masahiro Hashizume ${ }^{\mathrm{c}}$, Hisashi Kawai ${ }^{\mathrm{d}}$, Hirohiko Hirano ${ }^{\mathrm{d}}$, Motonaga Kojima $^{d}$, Yoshinori Fujiwara ${ }^{d}$, Shuichi Obuchi ${ }^{d}$, Mari Kogo $^{a}$, Masayuki Ohbayashi $^{\mathrm{a}}$, ${ }^{\text {, Noriko }}$ Koyama ${ }^{a}$, Miki Takenaka ${ }^{a}$, Kazushige Ihara ${ }^{e}$

${ }^{a}$ Department of Pharmaceutical Therapeutics, Division of Clinical Pharmacy, Pharmacy School, Showa University, 1-5-8 Hatanodai, Shinagawa-ku, Tokyo 142-8555, Japan.

${ }^{b}$ Showa University Research Institute for Sports and Exercise Science, 2-1-1 Fujigaoka, Aoba-ku, Yokohama 227-5818, Japan. ${ }^{c}$ Department of Psychosomatic Medicine, School of Medicine, Toho University, 5-21-16 Oomori-nishi, Oota-ku, Tokyo 143850, Japan.

${ }^{d}$ Tokyo Metropolitan Institute of Gerontology; 35-2 Sakae-cho, Itabashi-ku, Tokyo 173-0015, Japan.

${ }^{e}$ Department of Social Medicine, Hirosaki University Graduate School of Medicine, 1 Bunkyou-chou, Hirosaki City, Aomori Pref. 036-8560, Japan.

\begin{abstract}
Background: Frailty, which is losing body weight or lean body mass, experiencing fatigue and loss of strength or endurance, can easily result in falls and fractures with hospitalization and bedridden conditions. Brain-derived neurotrophic factor (BDNF), which is involved in neurogenesis, phenotypic differentiation, and neuronal survival, is gaining traction in the field of gerontology research as a relevant factor in cognition and vitality in the older population. We studied a distribution of mean BDNF levels or hand grip strengths according to BMI classification, and found the lowest BMI class of thin category had significantly $(P<0.01)$ lower mean BDNF level or mean hand grip strength than in the normal or obese 1 category of BMI. Therefore we defined the lowest BMI category "thin" as "prodromal frailty", but not pre-frailty. We report the findings of "prodromal frailty" and discuss how to prevent this stage from advancing to frailty.

Methods: We measured serum BDNF concentrations, BMI and various health parameters in 805 elderly (aged 65-84) regional health examination participants from the Tokyo area. Their serum BDNF levels and grip strengths were divided according to BMI classification.

Results: The mean serum BDNF levels increased linearly according to BMI categorization from thin $(<18.4$ $\left.\mathrm{kg} / \mathrm{m}^{2}\right)$ to obese $2\left(30-34.9 \mathrm{~kg} / \mathrm{m}^{2}\right)$ and decreased in the obese 3 group $\left(35-39.9 \mathrm{~kg} / \mathrm{m}^{2}\right)$. The mean serum BDNF level in the thin category was significantly lower than that in the normal category $(P<0.01)$. The BDNF levels in the obese 1 and obese 2 categories did not differ significantly from that in the normal category. Mean hand grip strength $(\mathrm{kg})$ was also similarly distributed to the mean BDNF level distribution in the classified BMIs.

Conclusion: Older people in the thin BMI category had significantly lower serum BDNF levels and weaker hand grip strength than those in the normal or slightly obese categories, placing them in the conditon of "prodromal frailty" but not pre-frailty.
\end{abstract}

Keywords: Frailty, body mass index, brain-derived neurotrophic factor, health examination, hand grip strength

* Corresponding author: Mitsugu Hachisu

Mailing address: Department of Pharmaceutical Therapeutics, Division of Clinical Pharmacy, Pharmacy School, Showa University, 1-5-8 Hatanodai, Shinagawa-ku, Tokyo 142-8555, Japan.

Email: mhachisu@pharm.showa-u.ac.jp

Received: 24 July 2020 / Accepted: 22 Septemper 2020

\section{Introduction}

Brain-derived neurotrophic factor (BDNF) which plays a role in neurogenesis, phenotypic differentiation, and neuronal survival, is reported to be related to depression and Alzheimer's disease [1-4]. It was found that serum BDNF 
level increases after exercise, and the repeated exercise enhances mood or cognitive functions in elderly people as well as mild cognitive impairment (MCI) patients [5]. Therefore, BDNF is gaining traction in the field of gerontology research and is considered relevant to the vitality of individuals. Older people who are losing weight or have low body mass reported fatigue, loss of strength and endurance. Fried et al. [6] defined frailty, in a study of more than 5,000 US community elderly people, as fulfilling three of the five following criteria; 1) lowering body weight, 2) weakness shown as reduced hand grip strength, 3) poor endurance and energy, 4) slowness, evidenced by slow gait speed and performance, and 5) low physical activity levels [6]. Once older people become frail they easily fall and fracture bones, which results in hospitalization and then becoming bedridden. Furthermore, frailty increases the risk of morbidity for various other diseases, including psychiatric problems such as deterioration of cognition and depression [7]. Ingres et al. [8] and Coelho et al. [9] reported association of frailty with low serum BDNF levels and cognition deterioration and they noted that interventions such as exercise improved symptoms of both frailty and cognition deterioration.

In our previous study, we reported a relationship between serum BDNF level and BMI in elderly people using a regression analysis [10]. In this report, we discovered significantly lower BDNF levels and weaker hand grip strength in one BMI category compared to that of others. These categories were classified according to the Japanese Society for the Study of Obesity (JASSO). Although our cohort contained healthy subjects, who were invited to participate in a medical examination, we found there were some "prodromal frailty", but not pre-frailty in the cohort. Therefore, we report the occurrence of "prodromal frailty" in the healthy subjects and discuss measures that can prevent the shift from "prodromal frailty" stage to frailty in these subjects, with respect to serum BDNF levels.

The demographic data and their relation to serum BDNF levels are as follows [10]: the mean participant age was 73.2 (SD: 4.9) years and the mean serum BDNF level was 9.40 (SD: 4.06$) \mathrm{ng} / \mathrm{mL}(n=805)$ among those $65-84$ years of age with distributing normally and was no sex difference. Individuals with cardiovascular disease represented $16.4 \%$ of the cohort and their BDNF levels were significantly lower than that of people without cardiovascular disease. Hypertension, hyperlipidemia, and diabetes mellitus was reported in $45.5 \%, 30.7 \%$, and $11.8 \%$, of the cohort, respectively. The serum BDNF levels of these participants did not differ from that in healthy people. The use of medicines such as anti-hypertensive drugs, anti-inflammatory drugs, hypnotics, anxiolytics, and osteoporosis drugs was reported in $43.6 \%, 9.2 \%, 14.0 \%, 7.7 \%$, and $12.0 \%$ of participants, respectively. The serum BDNF levels in subjects taking hypnotics for less than 1 year were significantly lower and significantly higher in subjects taking hypnotics for more than 1 year than in subjects not taking hypnotics. The serum BDNF levels in participants taking medicines other than hypnotics were not different from participants taking no medicines.

\section{Methods}

\section{Participants}

Participants undergoing medical health examinations were recruited by the Tokyo Metropolitan Institute of Gerontology as previously described [10]. Briefly, we sent a letter to 7,162 community-dwelling elderly individuals aged 65-84 years to invite them to participate in a medical health examination in the Tokyo area; of these 805 participants were willing to undergo the examination. We used a "yes" or "no" questionnaire to investigate past diseases, and the use of medicines was classified into the following categories: "taking no medicines", "taken within one year", and "taken for more than one year".

We obtained informed consent to perform the medical health examination, including blood collection, following oral explanations provided before the examination. Subjects with low basic activities of daily living (ADL) based on Katz Index under 3 points, severe visual and auditory disorders, severe post-stroke symptoms, and cognitive impairment with MMSE (Mini-mental state examination) scores under 24 were excluded [10].

We assert that all procedures contributing to this study complied with the ethical standards of the relevant national committees on human experimentation. The study was conducted in accordance with the Declaration of Helsinki (as revised in Brazil 2013), and the protocol was approved by the Ethics committee of the Showa University School of Pharmacy (Approval No. 160, August 4, 2012).

\section{Measurements of BMI, \%BFM, and hand grip strength}

Total body fat mass was measured using a Well-scan multi-frequency bioelectrical impedance analyzer (Elk Corporation, Japan) and expressed as \%BFM. BMI was calculated as body weight $(\mathrm{kg}) /$ height $\left(\mathrm{m}^{2}\right)$. Handgrip strength was measured once in each hand using a Smedley grip dynamometer (As One, Osaka, Japan) before the blood withdrawing and the higher value was incorporated for the data.

\section{Measurement of serum BDNF concentrations}

Blood was drawn at the end of health examinations and was centrifuged $1,500 \mathrm{x}$, at 4 degrees centigrade for $15 \mathrm{~min}$. The sera were transferred to a new set of polyethylene tubes and stored at -80 degrees centigrade until measurement. The serum BDNF levels were measured by an enzyme-linked immunosorbent assay using the BDNF Emax immunoassay system (Promega Corp., Madison, WI, USA.) according to the supplier protocol [10]. All samples were assayed in duplicate.

\section{Statistical analysis}

We used Student's $t$-tests to compare hand grip strength 
between male and female, and an analysis of variance (ANOVA) to compare BDNF levels across BMI or \%BFM categories and hand grip strengths across BMI categories. Then, the BDNF level or hand grip strength in each BMI class was analyzed with multiple comparisons using the Bonferroni correction. The differences were considered statistically significantly when they had $P<0.05$. We used PASW Statistics for Windows, version 18.0 to perform all statistical analyses (SPSS Inc., Chicago, IL, USA).

\section{Results}

\section{Serum mean BDNF levels in each BMI classification}

We re-analyzed the distribution of mean serum BDNF levels according to BMI classification. The BMI was classified as thin $\left(<18.4 \mathrm{~kg} / \mathrm{m}^{2}\right)$, normal $(18.5-24.9 \mathrm{~kg} /$ $\left.\mathrm{m}^{2}\right)$, obese $1\left(25.0-29.9 \mathrm{~kg} / \mathrm{m}^{2}\right)$, obese $2(30.0-34.9 \mathrm{~kg} /$ $\mathrm{m}^{2}$ ), obese $3\left(35-39.9 \mathrm{~kg} / \mathrm{m}^{2}\right)$ and obese 4 (over $40 \mathrm{~kg} / \mathrm{m}^{2}$ ) based on the JASSO classification [11]. The distribution of subjects among the BMI categories is summarized in Table 1 . The mean serum BDNF levels increased linearly from thin to obese 2, then decrease in the obese 3 category (Figure 1). The mean serum BDNF level in the normal category was significantly $(P<0.01)$ higher than that in the thin category, while it is not significant from obese 1 category or obese 2 category, respectively (Figure 1). Moreover, the BDNF levels in the thin category were also significantly lower than those in the obese $1(P<0.01)$ and obese $2(P<0.05)$ categories.

\section{Serum mean BDNF levels in \%BFM classifications}

We analyzed the distribution of mean serum BDNF concentrations across \%BFM categories. \%BFM was classified according to $5 \%$ point intervals from below $14.9 \%$ to over $40 \%$ using a modified $\%$ BFM chart de-

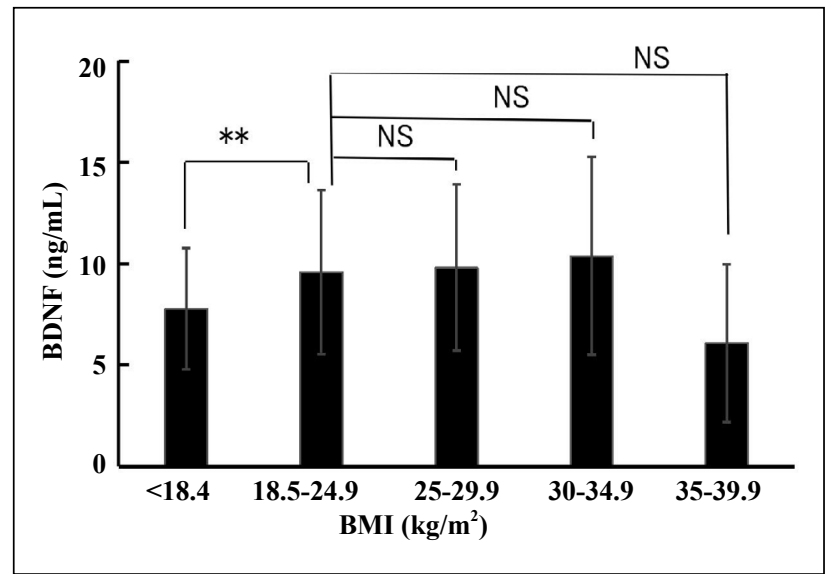

Figure 1. Serum BDNF levels in each BMI classification. The BDNF levels in each BMI category were expressed mean \pm SD and analyzed by ANOVA (Levene's test $F$ value $2.1783, P=0.0697$ ). Multiple comparison was performed using the Bonferroni correction. The data is expressed when compared with the normal BMI category of $18.5-24.9 \mathrm{~kg}$ / $\mathrm{m}^{2}$. ** $P<0.01$, NS: no significant. BDNF: brain-derived neurotrophic factor, BMI: body mass index.
Table 1. Distribution of subjects depending on BMI classification. The BMI classification and the category are followed by Japanese Society for the Study of Obesity [11].

\begin{tabular}{|c|c|c|}
\hline BMI classification & Category & Number of subjects (\%) \\
\hline$<18.4$ & thin & $67 \quad(8.3 \%)$ \\
\hline $18.5-24.9$ & normal & $548(68.1 \%)$ \\
\hline $25-29.9$ & obese 1 & $165(20.5 \%)$ \\
\hline $30-34.9$ & obese 2 & $20(2.5 \%)$ \\
\hline $35-39.9$ & obese 3 & $(0.6 \%)$ \\
\hline$>40$ & obese 4 & $(0 \%)$ \\
\hline
\end{tabular}

Table 2. Distribution of subjects depending on BMI classification. The $\% \mathrm{BFM}$ was classified with the $5 \%$ point of steps, and it is categorized by the modified \%BFM chart described by Tanita [12].

\begin{tabular}{llc}
\hline BMI classification & Category & Number of subjects (\%) \\
\hline$<14.9$ & thin & $24(3.0 \%)$ \\
$15-19.9$ & standard [-] & $68(8.5 \%)$ \\
$20-24.9$ & standard $[+]$ & $176(21.8 \%)$ \\
$25-29.9$ & pre-obese & $244(30.3 \%)$ \\
$30-34.9$ & obese i & $201(25.0 \%)$ \\
$35-39.9$ & obese ii & $78(9.7 \%)$ \\
$>40$ & obese iii & $14(1.7 \%)$ \\
\hline
\end{tabular}

scribed by Tanita [12]. The distribution of subjects based on \%BFM classification is shown in Table 2. The mean $\%$ BFM values did not differ between men and women in our older cohort $(27.7 \pm 7.4 \%[n=304]$ and $27.2 \pm 6.2$ $\%[n=501]$, respectively). As the mean \pm SD \%BFM of males and females was almost the same in the present cohort, we employed the modified Tanita's classification of \%BFM for male aged 60 and higher [12] to categorize the cohort irrespective of gender. The mean serum BDNF levels in each \%BFM category increased linearly from under $14.9 \% \mathrm{BFM}$ to over $40 \% \mathrm{BFM}$. However, the mean serum BDNF level in the under $14.9 \%$ BFM category $(7.6 \pm 3.0 \mathrm{ng} / \mathrm{mL})$ was not significantly different from that in the other \%BFM categories (Figure 2).

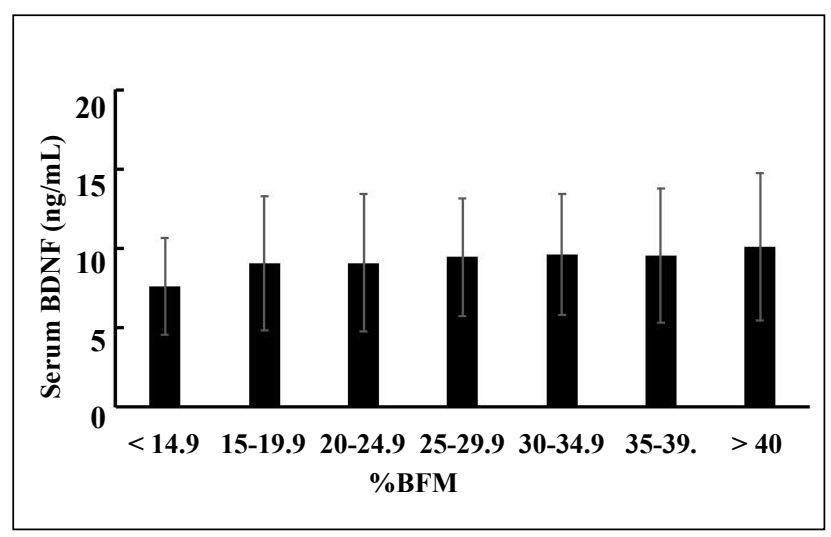

Figure 2. Serum BDNF levels at \%BFM classifications at $5 \%$ steps from $<\mathbf{1 4 . 9 \%}$ to $>\mathbf{4 0 . 0}$. The BDNF levels in each \%BFM category were expressed as mean \pm SD and analyzed by ANOVA (Levene's test $F$ value $0.5393, P=0.7785)$. Multiple comparison was performed using the Bonferroni correction. There was no significance when compared with the $\%$ BFM of $<14.9 \%$. 


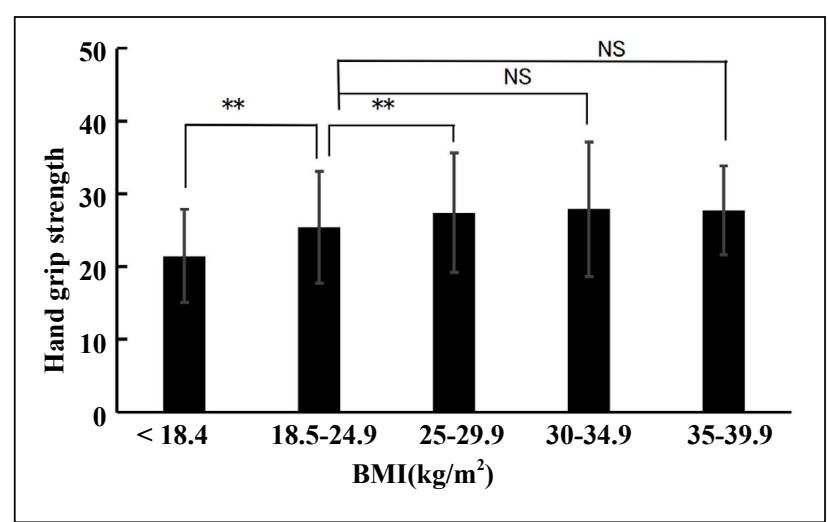

Figure 3. Handgrip strengths (kg) in each BMI classification. The hand grip strengths in each BMI category were expressed mean \pm SD and analyzed by ANOVA (Levene's test $F$ value 3.2310, $P=0.01$ ). Multiple comparison was performed using the Bonferroni correction. The data is expressed when compared with the normal BMI category of 18.5-24.9 $\mathrm{kg} / \mathrm{m}^{2}$. $* * P<0.01, * P<0.05$, NS: no significant. BDNF: brain-derived neurotrophic factor, BMI: body mass index.

\section{Hand grip strength in each classified BMI}

The mean hand grip strength in our cohort was $25.5 \pm 7.84$ $\mathrm{kg}$, exhibiting a significant difference $(P<0.01)$ between males $(32.1 \pm 6.99 \mathrm{~kg})$ and females $(21.21 \pm 4.75 \mathrm{~kg})$. The distributions of mean hand grip strengths in the classified BMI category from thin to obese 3 are shown in Figure 3 . The mean hand grip strength in the thin BMI category $(21.4 \pm 6.4 \mathrm{~kg})$ was significantly $(P<0.01)$ lower than that in the normal BMI $(25.4 \pm 5.4 \mathrm{~kg})$, obese 1 and obese 2 categories. Further, the mean hand grip strength in the normal BMI category was significantly lower than that in the obese 1 category $(P<0.05)$. Hence, hand grip strength was lowest in the thin BMI category (Figure 3 ).

\section{Discussion}

The mean \pm SD BMI in this cohort of community-dwelling individuals in the Tokyo area was $22.8 \pm 3.4 \mathrm{~kg} / \mathrm{m}^{2}$ $\left(23.1 \pm 3.1 \mathrm{~kg} / \mathrm{m}^{2}\right.$ in male and $22.6 \pm 3.2 \mathrm{~kg} / \mathrm{m}^{2}$ in female participants). The reported BMIs among communitydwelling individuals aged 60 years and over in rural and urban areas of Indonesia were $22.7 \pm 2.2$ and $24.9 \pm 3.4$ $\mathrm{kg} / \mathrm{m}^{2}$, respectively [13]. In the Programa Municipal da Terceira Idade (PMTI) program in Vicosa, Minas Gerais, Brazil, the mean BMI was $27.4 \mathrm{~kg} / \mathrm{m}^{2}$ among those aged 60 years and over (average 72 years), and about half were overweight [14]. The BMI in the current cohort of community-dwelling individuals in the Tokyo area was close to that in the rural area of Indonesia and lower than that in Brazil. Assessment of the distributions of the participants by BMI classification showed that $68.1 \%$ and $8.3 \%$ were the normal and thin categories, respectively, among those aged 65-84 years in the Tokyo area (Table 1). Among 12,544 participants of the US National Health and Nutrition Examination Survey (NHANES) aged over 20 years, $1.4 \%$ and $30.6 \%$ were in the thin and normal BMI categories [15], proportions $1 / 6$ and $1 / 2$ those in our cohort, respectively.

The mean \pm SD $\%$ BFM in our cohort was $27.4 \pm 6.3 \%$ (male $27.7 \pm 6.4 \%$ and female $27.2 \pm 6.2 \%$ ), and was comparable between males and females. This cohort is unique as the $\% \mathrm{BFM}$ in females is generally higher than that in males $[12,13]$. The $\% \mathrm{BFM}$ is increased with age in males and remained steady in females; therefore, the values were comparable between sexes [16].

In this study, the mean serum BDNF levels in classified BMI increased linearly from the thin to obese 2 BMI categories and decrease in the obese 3 category. Unexpectedly, the mean serum BDNF level in the thin category was significantly lower than that in the normal BMI category $(P$ $<0.01)$. However the mean BDNF level in normal BMI is not significantly different from that in obese 1 , obese 2 and obese 3 , respectively.

Nanri et al. [17] reported results that were similar to our findings, they reported that the mean serum BDNF levels in the BMI categories from $<21.0$ to $>27.0$ showed significant positive correlation in Japanese participants aged 18-70. Unfortunately, there was no classification of thin $(<18.4)$ BMI category in this cohort. Moreover, findings consistent with our data on the positive correlation of serum BDNF and BMI were reported by Golden et al. [18] in older women $(70.3 \pm 0.76$ years old $)$ from the US and by Suwa et al. [19] in Japanese women with type 2 diabetes mellitus aged 34-59 years; however there were also no classification of thin BMI category in these reports. On the other hand, no correlation between plasma BDNF levels and BMI was reported in older people aged 60-81 years from the US [20].

With respect to \%BFM and serum BDNF levels, a positive relationship was reported by the regression analysis in our previous report [10]. When we categorized the $\% \mathrm{BFM}$ at $5 \%$ point interval from under $14.9 \%$ to over $40 \%$, the mean BDNF level in the $<14.9 \%$ BFM category was not significantly different from that of the other categories. The accumulation of BFM influences various metabolic activities, especially obesity. Obesity tends to be insulinresistant with the development of low-grade inflammation, including an increase in inflammatory cytokines such as interleukin 6 (IL-6) [21]. Inflammatory cytokines inhibit BDNF expression, thereby decreasing serum BDNF level [22]. Moreover, it has been reported that the circulating BDNF levels are decreased in patients with diabetes mellitus showing symptoms of nephropathy and retinopathy [23, 24]. Since Keys et al. [25] reported that there is a strong correlation between BMI and BFM, BMI is currently used as an index of obesity. Indeed, BMI was also well correlated with \%BFM $(r=0.9912, P=0.01)$ in our cohort, while there are some difference in the distribution pattern of mean BDNF levels in the BMI classification and those in \%BFM classification as shown at results. Low BMI or BFM indicates thinness while low BMI indicates not only low BFM but also low muscle mass, as BMI is calculated as the body weight $(\mathrm{kg})$ divided by the square of the height $\left(\mathrm{m}^{2}\right)$. Muscle mass and BDNF levels are positively correlated [26], while increased BFM de- 
creased BDNF levels through inflammation, as described above. Therefore, it can be interpreted by the previous logic that the BDNF level in the lowest BMI of the thin category was significantly lower than that in normal, obese 1 and obese 2 categories, while the BDNF level in the lowest \%BFM category was not significantly different from other categories of \%BFM.

Low muscle mass may cause sarcopenia and frailty. We observed significantly lower serum BDNF levels in the thin BMI category than that in the normal BMI category. Frailty is a condition characterized by weakness with low body weight and low activity that is sometimes also reported to include reduced mood and cognition [7]. Ingles et al. [8] reported lower plasma BDNF levels in frail individuals than those non-frail individuals, which was associated with lower cognition.

We observed significantly lower hand grip strength in the thin BMI category than that in the normal, obese 1 , and obese 2 BMI categories. This pattern was similar to the distribution observed for serum BDNF levels according to BMI classification. The five frailty criteria proposed by Fried et al include "weakness: reduced hand grip strength" [6]. The thin BMI category showed low serum BDNF levels and weak hand grip strength. Moreover, individuals with frailty reportedly show low levels of circulating BDNF [8]. Therefore, individuals in the thin BMI category with low BDNF levels and weak hand grip strength in our cohort likely corresponded to "prodromal frailty" but not pre-frailty.

We previously reported higher serum BDNF concentrations in individuals with thick quadriceps muscles [10]. These individuals may receive adequate physical exercise in their daily lives. Physical exercise reportedly increases serum BDNF levels in both pre-frail and non-frail women [27]. Moreover, the authors suggested that BDNF levels may be a key pathophysiological mediator in frailty. Therefore, we first showed the presence of "prodromal frailty" in a healthy cohort of community-dwelling individuals with low BDNF levels, weak handgrip strength, and low BMI. Individuals in the thin category with low BDNF levels should start exercising and ingest proper nutrition with high protein levels to prevent frailty. Because, it is reported that the older adults with higher muscle mass showed a low mortality risk in a 10-16-year longitudinal follow-up study in the US [28]. Therefore, resistance training, especially hypertrophied resistance training, is recommended to increase muscle mass [29-31] at 8 to 12 reps per set for more than three sets [29]. Leg exercises; i.e., squats, are recommended for hypertrophied thigh muscle, one of the largest muscles in the body, for prevention of fall and slowed gait speed associated with frailty. Consumption of high protein and/or amino acid diets reverse frailty and increase strength and muscle mass with elevated levels of circulating BDNF [32].

There are some limitations in this study. The cohort was comprised of individuals who were interested in and paying attention to their health, who presented to the institute at their own volition and mostly by themselves. Therefore, the cohort may be representative of vital older people rather than average community-dwelling older individuals aged 65-84 years in the Tokyo area. Moreover, the observations were made on a single day rather than as part of a longitudinal study; therefore, BMI or \%BFM changes in individuals do not explain their changes in BDNF level. BMI is a well-known assessment of the level of fat (obese) in the human body and it is calculated by an equation [body weight $(\mathrm{kg})$ / height $\left(\mathrm{m}^{2}\right)$ ], therefore it also includes muscle mass. Therefore, we cannot conclusively show that the presence of "prodromal frailty" in the thin category of BMI depends on the level of muscle mass or BFM. However, due to the ease of calculating BMI, it was employed to analyze the presence of "prodromal frailty" in a large cohort.

In summary, older people in the thin BMI category had significantly lower serum BDNF levels and weaker hand grip strength than those in the normal or slightly obese categories. The thin category with findings of low BDNF levels and weak hand grip strength was designated as "prodromal frailty".

\section{Declarations}

Acknowledgements: We acknowledge support for this study from a Health and Labor Sciences Research Grant (H23-Choju-Ippan-001, H23-Choju-Ippan-002) and a JSPS KAKENHI research Grant (grant number: 21590717). We would like to thank Editage (www.editage.com) for English language editing.

Authors' Contributions: Conceptualization, M.H. and K.I.; data curation, H.K.; formal analysis, M.H. and M.H.; investigation, M.H., M.H., H.K., H.H., M.K., Y.F., S.O., M.K., M.O., N.K., M.T., and K.I.; project administration, H.K., H.H., M.K., Y.F., S.O., and K.I.; resources, S.O. and K.I.; writing-original draft, M.H.; writing-review \& editing, M.H., M.K., M.O., N.K., M.T. and K.I. All authors have read and agreed to the published version of the manuscript.

Financial supports: We received support for this study from a Health and Labor Sciences Research Grant (H23Choju-Ippan-001, H23-Choju-Ippan-002) and a JSPS KAKENHI research Grant (grant number: 21590717).

Conflicts of Interest: The authors declare no conflict of interest.

Ethical approval and informed consent: We assert that all procedures contributing to this study complied with the ethical standards of the relevant national committees on human experimentation. The study was conducted in accordance with the Declaration of Helsinki (as revised in Brazil 2013), and the protocol was approved by the Ethics committee of the Showa University School of Pharmacy (Approval No. 160, August 4, 2012). We obtained informed consent to perform the medical health examination, including blood collection, following oral explanations provided before the examination. 


\section{References}

1. Ihara K, Yoshida H, Jones P B, et al. Serum BDNF levels before and after the development of mood disorders: a case-control study in a population cohort. Translational psychiatry, 2016, 6(4): e782-e782.

2. Yoshimura R, Mitoma M, Sugita A, et al. Effects of paroxetine or milnacipran on serum brain-derived neurotrophic factor in depressed patients. Progress in NeuroPsychopharmacology and Biological Psychiatry, 2007, 31(5): 1034-1037.

3. Leyhe T, Stransky E, Eschweiler G W, et al. Increase of BDNF serum concentration during donepezil treatment of patients with early Alzheimer's disease. European Archives of Psychiatry and Clinical Neuroscience, 2008, 258(2): 124-128.

4. Duman R S. Synaptic plasticity and mood disorders. Molecular psychiatry, 2002, 7(1): S29-S34.

5. Anderson-Hanley C, Barcelos N M, Zimmerman E A, et al. The aerobic and cognitive exercise study (ACES) for community-dwelling older adults with or at-risk for mild cognitive impairment (MCI): neuropsychological, neurobiological and neuroimaging outcomes of a randomized clinical trial. Frontiers in aging neuroscience, 2018, 10: 76.

6. Fried L P, Tangen C M, Walston J, et al. Frailty in older adults: evidence for a phenotype. The Journals of Gerontology Series A: Biological Sciences and Medical Sciences, 2001, 56(3): M146-M157.

7. Albala C, Lera L, Sanchez H, et al. Frequency of frailty and its association with cognitive status and survival in older Chileans. Clinical interventions in aging, 2017, 12: 995.

8. Inglés M, Gambini J, Mas-Bargues C, et al. Brain-derived neurotrophic factor as a marker of cognitive frailty. The Journals of Gerontology: Series A, 2017, 72(3): 450-451.

9. Coelho F M, Pereira D S, Lustosa L P, et al. Physical therapy intervention (PTI) increases plasma brain-derived neurotrophic factor (BDNF) levels in non-frail and prefrail elderly women. Archives of gerontology and geriatrics, 2012, 54(3): 415-420.

10. Hachisu M, Hashizume M, Kawai H, et al. Relationships between serum brain-derived neurotrophic factor concentration and parameters for health scores in community-dwelling older adults[J]. Geriatrics \& gerontology international, 2018, 18(3): 456-461.

11. Ministry of Health, Labor and Welfare Health information site for prevention of lifestyle-related diseases on December 17, 2019. Available online: https://www.ehealthnet.mhlw.go.jp/information/food/e-02-001.html

12. TANITA Worldwide. How to read the measurement items of the body composition monitor. 2020. Available online: https://www.tanita.co.jp/health/measure/taisoseikei/

13. Hanindriyo L, Widita $E$, Widyaningrum $R$, et al. Influence of residential characteristics on the association between the oral health status and BMI of older adults in Indonesia. Gerodontology, 2018, 35(3): 268-275.

14. Gonçalves D F, Tinoco A L A, Ribeiro R C L, et al. Nutri- tional status and epidemiological profile of elderly people. Archives of gerontology and geriatrics, 2012, 55(1): 1-4.

15. Abramowitz M K, Hall C B, Amodu A, et al. Muscle mass, BMI, and mortality among adults in the United States: A population-based cohort study. PloS one, 2018, 13(4): e0194697.

16. Campisi J, Finn K E, Bravo Y, et al. Sex and age-related differences in perceived, desired and measured percentage body fat among adults. Journal of Human Nutrition and Dietetics, 2015, 28(5): 486-492.

17. Nanri A, Kochi T, Eguchi M, et al. Demographic and lifestyle correlates of brain-derived neurotrophic factor in a working population: The Furukawa Nutrition and Health Study. Psychiatry research, 2019, 272: 581-586.

18. Golden E, Emiliano A, Maudsley S, et al. Circulating brain-derived neurotrophic factor and indices of metabolic and cardiovascular health: data from the Baltimore Longitudinal Study of Aging. PLoS one, 2010, 5(4): e10099.

19. Suwa M, Kishimoto H, Nofuji Y, et al. Serum brainderived neurotrophic factor level is increased and associated with obesity in newly diagnosed female patients with type 2 diabetes mellitus. Metabolism, 2006, 55(7): 852-857.

20. Pillai A, Bruno D, Sarreal A S, et al. Plasma BDNF levels vary in relation to body weight in females[J]. PLoS One, 2012, 7(7): e39358.

21. Mauer J, Chaurasia B, Goldau J, et al. Signaling by IL-6 promotes alternative activation of macrophages to limit endotoxemia and obesity-associated resistance to insulin. Nature Immunology, 2014, 15: 423-430.

22. Dhanda S, Gupta S, Halder A, et al. Systemic inflammation without gliosis mediates cognitive deficits through impaired BDNF expression in bile duct ligation model of hepatic encephalopathy. Brain, behavior, and immunity, 2018, 70: 214-232.

23. Kurajoh M, Kadoya M, Morimoto A, et al. Plasma brainderived neurotrophic factor concentration is a predictor of chronic kidney disease in patients with cardiovascular risk factors-Hyogo Sleep Cardio-Autonomic Atherosclerosis study. Plos one, 2017, 12(6): e0178686.

24. Guo M, Liu H, Li S, et al. Low serum brain-derived neurotrophic factor but not brain-derived neurotrophic factor gene Val66met polymorphism is associated with diabetic retinopathy in Chinese type 2 diabetic patients. Retina, 2017, 37(2): 350-358.

25. Keys A, Fidanza F, Karvonen M J, et al. Indices of relative weight and obesity. Journal of chronic diseases, 1972, 25(6-7): 329-343.

26. Máderová D, Krumpolec P, Slobodová L, et al. Acute and regular exercise distinctly modulate serum, plasma and skeletal muscle BDNF in the elderly. Neuropeptides, 2019, 78: 101961.

27. Coelho F M, Pereira D S, Lustosa L P, et al. Physical therapy intervention (PTI) increases plasma brain-derived neurotrophic factor (BDNF) levels in non-frail and prefrail elderly women. Archives of gerontology and geriat- 
rics, 2012, 54(3): 415-420.

28. Srikanthan P, Karlamangla A S. Muscle mass index as a predictor of longevity in older adults. The American journal of medicine, 2014, 127(6): 547-553.

29. Marston K J, Newton M J, Brown B M, et al. Intense resistance exercise increases peripheral brain-derived neurotrophic factor. Journal of science and medicine in sport, 2017, 20(10): 899-903.

30. Dinoff A, Herrmann N, Swardfager W, et al. The effect of exercise training on resting concentrations of periph- eral brain-derived neurotrophic factor (BDNF): a metaanalysis. PLoS one, 2016, 11(9): e0163037.

31. Marston K J, Newton M J, Brown B M, et al. Intense resistance exercise increases peripheral brain-derived neurotrophic factor. Journal of science and medicine in sport, 2017, 20(10): 899-903.

32. Lorenzo-López, L., Maseda, A., de Labra, C., RegueiroFolgueira, L., Rodríguez-Villamil, J. L., \& Millán-Calenti, J. C. (2017). Nutritional determinants of frailty in older adults: a systematic review. BMC geriatrics, 17(1), 108.

Cite this article as: Mitsugu $\mathrm{H}$, Masahiro $\mathrm{H}$, Hisashi $\mathrm{K}$, et al. Finding prodromal frailty in a communitydwelling healthy older cohort by survey of BDNF or hand grip strength classified by BMI. Aging Pathobiology and Therapeutics, 2020, 2(3): 155-161. 